\title{
Accidental Electric Shock during Pregnancy: Reflection on a Case
}

\author{
Johnny Awwad, MD ${ }^{1}$ Antoine Hannoun, MD ${ }^{1}$ Farah Fares, $\mathrm{BS}^{1} \quad$ Ghina Ghazeeri, MD ${ }^{1}$
}

\author{
${ }^{1}$ Department of Obstetrics and Gynecology, American University of \\ Beirut Medical Center, Beirut, Lebanon
}

Am J Perinatol Rep 2013;3:103-104.
Address for correspondence Ghina Ghazeeri, MD, Department of Obstetrics and Gynecology, American University of Beirut Medical Center, P.O. Box 113-6044, Beirut, Lebanon (e-mail: gg02@aub.edu.lb).

\begin{abstract}
Keywords

- accidental electric shock

- pregnancy

- fetal injury

- electrocution

Objectives Data on fetal effects following accidental electric shock during pregnancy are scarce. We report on a case of accidental maternal electric shock associated with benign fetal arrhythmia in a woman at 28 weeks' gestation.

Study Design Case report.

Results Although electrocution involving low-voltage, low-frequency current has been associated with fatal cardiac arrhythmias and conduction abnormalities, two protective parameters in the present case likely reduced the fetal injury: the dry skin at the site of current entry and the hand-to-hand pathway of current flow.

Conclusion Because the pathophysiology of electric injury is altered during pregnancy, assessment of fetal well-being should be prompted no matter how trivial an incident may appear.
\end{abstract}

The occurrence of accidental electric shock during pregnancy poses a dual challenge and is the source of significant concern with respect to maternal and fetal well-being. Data on fetal risks following accidental maternal electrocution are scattered. Fetal injuries have been reported to include sudden deaths, cardiac arrhythmias, intrauterine growth retardation, oligohydramnios, and abortion. ${ }^{1,2}$ Postmortem examinations have revealed dilatation of fetal cardiac chambers, as well as hemorrhages in fetal brain, kidneys, and liver. ${ }^{1}$ The most common source of published data on accidental maternal electrocution, however, has often been cumulative case reports, which suggest a fetal mortality of $76 \%{ }^{1,3}$ This type of reporting nevertheless is prone to significant overestimate bias, as it is often prompted by adverse fetal outcome. Although case reports have demonstrated an unrealistically high perception of fetal risks, a prospective cohort study from Toronto suggested that accidental electric shock during pregnancy poses major risks to the fetus only in $15 \%$ of cases and in the presence of particular enhancing parameters, ${ }^{4}$ such as the characteristics and pathway of the electric current.

We present a woman who sustained an accidental electric shock at 28 weeks into her gestation, resulting in fetal arrhythmia of benign and transient nature. Parameters believed to influence the extent and likelihood of fetal injury are also discussed.

\section{Case Report}

A 28-year-old primigravid woman presented at 28 weeks' gestation for accidental electric shock to her dry hand from a kitchen electric appliance with worn insulation connected to a $220-\mathrm{V}, 55-\mathrm{Hz}$ alternating current. The duration of the shock was estimated by the woman not to exceed 1 second, during which she reported hand tetany but no loss of consciousness. The woman reported to the clinic 2 hours later because of fetal hypoactivity and a remarkable change in fetal movement pattern, which became brisker and short-lasting.

Maternal physical examination revealed no signs of superficial burns or any current entry or exit skin marks. Because the woman was wearing rubber slippers, the flow of current was estimated to be hand-to-hand. Fetal Doppler monitoring showed an irregular fetal heart rhythm and missed beats, but no evidence of fetal distress. Ultrasound examination received

March 22, 2013

accepted after revision

May 10, 2013

published online

July 11, 2013
DOI http://dx.doi.org/

10.1055/s-0033-1348403. ISSN 2157-6998.
Copyright $\odot 2013$ by Thieme Medical Publishers, Inc., 333 Seventh Avenue, New York, NY 10001, USA. Tel: +1(212) 584-4662.
License terms

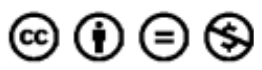


revealed a 1,220-g normal-looking male fetus with unremarkable amniotic fluid index and placentation.

The pregnant woman was monitored for 6 hours during which external fetal heart tracing reverted to regular rhythm and movements returned to previous normal pattern. She was discharged home and delivered at 38 weeks' gestation a live male newborn weighing 3,120 g with Apgar scores of 8 and 10 at 1 and 5 minutes, respectively. Postnatal physical examination was unremarkable with no burn skin marks identified. Electrocardiography revealed normal sinus rhythm and no functional conduction abnormalities. Echocardiography showed no particular structural abnormalities.

\section{Discussion}

Several parameters have been proposed to influence the seriousness of fetal effects following maternal electric shock. The characteristics of electricity and the pathway of current flow both appear to be important determinants of fetal risk during accidental maternal electrocution.

Alternating current seems to be associated with more serious risks than direct current, in that it can provoke repetitive tetanic muscle contractions causing the individual to grip the source of electricity leading to longer electric exposure. ${ }^{5}$ It is also associated with a ninefold greater risk of ventricular fibrillation than direct current, considering the same voltage and intensity parameters. ${ }^{5}$ Although a high-voltage electric source (1,000 Vor more) is usually the cause of a more harmful discharge of electricity, ${ }^{2}$ cardiac arrest from ventricular fibrillation and conduction failure appears to occur more commonly with low-voltage (less than 1,000 V), ${ }^{5}$ low-frequency (15 to $150 \mathrm{~Hz}$ ) alternating current. ${ }^{6}$ It was reported that $\sim 10$ to $46 \%$ of adult survivors experience subsequent cardiac arrhythmias, ${ }^{5}$ the most common of which are sinus tachycardia and premature ventricular systoles. Conduction abnormalities have also been described as a result of the vulnerability of the sinoatrial and atrioventricular nodes, ${ }^{7}$ and they are expressed as transient nonspecific ST-T-wave abnormalities, sinus bradycardia, and high-degree atrioventricular block on electrocardiography. ${ }^{8}$ Because very little is known about fetal arrhythmias occurring following accidental maternal electric shock, extrapolation from adult survivors' data may prove intuitive in that respect.

Dry maternal keratinized skin is highly resistant to electricity, hence playing the role of "gatekeeper" limiting the conduction of electric current to deeper structures. ${ }^{6}$ Moist skin, in contrast, conducts electricity more readily and therefore is associated with more extensive injury to internal organs despite often misleading superficial thermal injury and skin marks. The vertical hand-to-foot and head-to-foot pathways of current appear to carry the worst risk to the fetus as they travel through the uterus, placenta, and amniotic fluid. Keeping in mind that fetal skin is less resistant to the passage of electric current than mother's skin, it transmits current effectively, which increases the risk for fetal injury. ${ }^{4}$ In fact, fetal skin provides an excellent conduction platform and has been demonstrated to offer 200 times less resistance to the passage of current than postnatal skin. ${ }^{4}$ It has therefore been estimated that direct exposure to a 100 - to $380-\mathrm{V}, 25-$ $\mathrm{mA}$ current for 0.3 seconds could be lethal for the fetus. ${ }^{3}$ Fortunately, only $10 \%$ of home accidents involve such pathways. ${ }^{4}$ A horizontal hand-to-hand current flow represents a more common scenario and has been associated with least harm to the fetus albeit with a higher risk of maternal fatal cardiac arrhythmias. ${ }^{4}$

In the present case, the dry skin at the site of electric current entry and the horizontal hand-to-hand current flow appeared to have provided partial protection to the fetus, as evidenced by the immediate reversal of a benign pattern of fetal arrhythmia followed by uncomplicated term delivery with adequate fetal weight gain and excellent Apgar scores at birth.

Prior knowledge of the basic principles of physics may be helpful for treating physicians in interpreting the consequences of injuries sustained by the mother and her fetus following accidental electrocution during pregnancy. Particular attention should be provided to the nature of the electric current and its pathway during history taking. Low-voltage (less than $1,000 \mathrm{~V}$ ), low-frequency ( 15 to $150 \mathrm{~Hz}$ ) alternating current is a typical characteristic of home electrocution and has been associated with the highest risk for cardiac arrhythmias and conduction failure. Vertical current flow (hand-to-foot and head-to-foot) through a wet skin increases the frequency and propensity of fetal injury.

Because the pathophysiology of electric injury is altered during pregnancy, the consequences of electrocution in pregnant women may be more serious than actually anticipated. Fetal well-being should therefore be promptly assessed following maternal electric shock no matter how trivial an incident may appear.

\section{References}

1 Sornogyi E, Tedeschi CG. Injury by electrical force. In: Tedeschi CG, Eckert WG, Tedeschi LG, eds. Forensic Medicine. Philadelphia, PA: W.B. Saunders; 1977:645-676

2 Rosati P, Exacoustos C, Puggioni GF, Mancuso S. Growth retardation in pregnancy: experimental model in the rabbit employing electrically induced thermal placental injury. Int J Exp Pathol 1995;76:179-181

3 Fatovich DM. Electric shock in pregnancy. J Emerg Med 1993;11: 175-177

4 Einarson A, Bailey B, Inocencion G, Ormond K, Koren G. Accidental electric shock in pregnancy: a prospective cohort study. Am J Obstet Gynecol 1997;176:678-681

5 Spies C, Trohman RG. Narrative review: electrocution and lifethreatening electrical injuries. Ann Intern Med 2006;145:531-537

6 Jain S, Bandi V. Electrical and lightning injuries. Crit Care Clin 1999;15:319-331

7 James TN, Riddick L, Embry JH. Cardiac abnormalities demonstrated postmortem in four cases of accidental electrocution and their potential significance relative to nonfatal electrical injuries of the heart. Am Heart J 1990;120:143-157

8 Graber J, Ummenhofer W, Herion H. Lightning accident with eight victims: case report and brief review of the literature. J Trauma 1996;40:288-290 